\title{
A Well-Conditioned Time Domain EFIE for Densely Discretized Low Frequency Problems
}

\author{
Y. Beghein* $\quad$ K. Cools ${ }^{\dagger} \quad$ F.P. Andriulli ${ }^{\ddagger}$
}

\begin{abstract}
The time domain electric field integral equation (TD-EFIE) models transient scattering by perfect electric conductors. Upon discretization, this equation yields an ill-conditioned system matrix when the time step is large (low frequency breakdown), or the mesh is dense (dense discretization breakdown). Furthermore, its solution suffers from spurious static loop currents (DC instability). The quasi-Helmholtz projected TD-EFIE (qHP-TDEFIE) is an alternative formulation of the TD-EFIE which is immune to both low frequency breakdown and DC instability. In this contribution, a multiplicative Calderón preconditioner is constructed for the qHP-TDEFIE, which renders it immune to dense discretization breakdown. This ensures that transient electromagnetic scattering problems can be solved efficiently and accurately, even for slowly varying fields in the presence of small geometrical features.
\end{abstract}

\section{INTRODUCTION}

Transient scattering by perfect electric conductors is modeled by the time domain electric field integral equation (TD-EFIE). The standard technique to numerically solve the TD-EFIE, is to discretize it using a temporal collocation or Galerkin method. The resulting system can then be solved using the marching-on-in-time (MOT) algorithm.

This approach gives rise to ill-conditioned MOT system matrices when it is applied either to densely discretized geometries (dense discretization breakdown) or with large time steps (low frequency breakdown). Furthermore, the TD-EFIE suffers from DC instability: its numerical solution is plagued by spurious static loop currents.

Dense discretization breakdown can be cured using Calderón preconditioning [1]. The so-called dottrick Calderón preconditioned TD-EFIE [1] is furthermore free from low frequency breakdown as well as DC instability, but only when it is applied to simply connected geometries $[2,3]$.

Low frequency breakdown has previously been combated using loop-star or loop-tree decomposition [4]. This approach has two drawbacks. First, for multiply connected geometries, global topologi-

\footnotetext{
${ }^{*}$ Department of Information Technology (INTEC), Ghent University, Belgium, e-mail: yves.beghein@ugent.be.

$\dagger$ Electrical Systems and Optics Research Division, University of Nottingham, Nottingham NG7 2RD, U.K.

${ }_{\ddagger}$ Microwave Department of Telecom Bretagne - Institut Mines-Telecom, Brest, France.
}

cal loops must be detected, which is a computationally complex operation for a general mesh. Second, explicitly transforming the equation to the basis of loops and stars (or trees) leads to ill-conditioning when used in conjunction with Calderón preconditioning [5].

In [3], the quasi-Helmholtz projected TD-EFIE (qHP-TDEFIE) was introduced. By employing the projector operators defined in [6], the loop and star components of the TD-EFIE were separated without resorting to an explicit loop/star basis transformation. These components were then integrated or differentiated with respect to time, and separately discretized using temporal Galerkin procedures. This resulted in an equation that is immune to both low frequency breakdown and DC instability. The qHP-TDEFIE is applicable to both simply and multiply connected geometries, without requiring the detection of global topological loops.

In this contribution, a Calderón preconditioner is developed for the qHP-TDEFIE. This preconditioner allows the efficient simulation of scattering problems, even if they involve fine geometrical details or slowly varying incident fields.

\section{SPATIAL DISCRETIZATION}

\subsection{The TD-EFIE}

Consider a perfectly conducting body $\Omega$, with boundary $\Gamma$ and external normal vector $\hat{\boldsymbol{n}}$. When an electric field $\boldsymbol{e}^{i}$ illuminates $\Omega$ at $t>0$, a current $\boldsymbol{j}$ is induced on $\Gamma$, which satisfies the TD-EFIE:

$$
\eta(\mathcal{T} \boldsymbol{j})(\boldsymbol{r}, t)=-\hat{\boldsymbol{n}} \times \boldsymbol{e}^{i}(\boldsymbol{r}, t) \quad \forall \boldsymbol{r} \in \Gamma, t>0,
$$

where

$$
\begin{aligned}
(\mathcal{T} \boldsymbol{j})(\boldsymbol{r}, t) & =-\frac{1}{c} \hat{\boldsymbol{n}} \times \int_{\Gamma} \frac{\partial_{t} \boldsymbol{j}\left(\boldsymbol{r}^{\prime}, \tau\right)}{4 \pi R} d s^{\prime} \\
& +c \hat{\boldsymbol{n}} \times p . v . \int_{\Gamma} \operatorname{grad} \frac{\partial_{t}^{-1} \operatorname{div}_{\Gamma}^{\prime} \boldsymbol{j}\left(\boldsymbol{r}^{\prime}, \tau\right)}{4 \pi R} d s^{\prime},
\end{aligned}
$$

$\tau=t-R / c, \eta=\sqrt{\mu_{0} / \epsilon_{0}}, c=1 / \sqrt{\epsilon_{0} \mu_{0}}$ and $R=$ $\left|\boldsymbol{r}-\boldsymbol{r}^{\prime}\right|$. Further define $\partial_{t}^{-1} f(t)=\int_{-\infty}^{t} f(\tau) d \tau$.

The unknown current $\boldsymbol{j}(\boldsymbol{r}, t)$ is expanded in RWG basis functions $\boldsymbol{f}_{m}(\boldsymbol{r})[7]$

$$
\boldsymbol{j}(\boldsymbol{r}, t)=\sum_{m=1}^{N_{S}} \mathrm{j}_{m}(t) \boldsymbol{f}_{m}(\boldsymbol{r}) .
$$


Inserting this into (1) and testing the resulting equation with the rotated RWG functions $\hat{\boldsymbol{n}} \times$ $\boldsymbol{f}_{m}(\boldsymbol{r})$ yields

$$
(\mathcal{Z} \mathbf{j})(t)=-\mathrm{e}(t)
$$

where $\mathcal{Z}$ is an operator that maps a time-dependent RWG expansion coefficient vector into a timedependent RWG testing coefficient vector - see [3] for its definition.

Calderón preconditioning is achieved by discretizing the equation

$$
\eta^{2}\left(\mathcal{T}^{2} \boldsymbol{j}\right)(\boldsymbol{r}, t)=-\eta\left(\mathcal{T}\left(\hat{\boldsymbol{n}} \times \boldsymbol{e}^{i}\right)\right)(\boldsymbol{r}, t),
$$

resulting in

$$
\left(\tilde{\mathcal{Z}} \mathbf{G}_{\mathrm{mx}}^{-1} \mathcal{Z} \mathbf{j}\right)(t)=-\left(\tilde{\mathcal{Z}} \mathbf{G}_{\mathrm{mx}}^{-1} \mathrm{e}\right)(t) .
$$

In this, $\tilde{\mathcal{Z}}$ is obtained by discretizing the $\mathcal{T}$ operator using the Buffa-Christiansen (BC) functions $\boldsymbol{g}_{m}(\boldsymbol{r})$ [8] rather than the RWG functions $\boldsymbol{f}_{m}(\boldsymbol{r})$. The Gram matrix $\mathbf{G}$ is defined as

$$
\left[\mathbf{G}_{\mathrm{mx}}\right]_{m n}=\int_{\Gamma}\left(\hat{\boldsymbol{n}} \times \boldsymbol{f}_{m}(\boldsymbol{r})\right) \cdot \boldsymbol{g}(\boldsymbol{r}) d s .
$$

Both (3) and (5) are discrete in space, but continuous in time. In order to construct a numerical solution, they need to be discretized in time. However, they will first be further manipulated in order to obtain a low frequency and DC stable scheme.

\subsection{Rescaling}

Define the auxiliary unknown

$$
\mathrm{y}(t)=\left(\mathbf{P}^{\Lambda H}+\partial_{t}^{-1} \mathbf{P}^{\Sigma}\right) j(t)
$$

satisfying

$$
\begin{gathered}
\left(\mathcal{Z}^{\prime} \mathbf{y}\right)(t)=-\left(\partial_{t}^{-1} \mathbf{P}^{\Lambda H}+\mathbf{P}^{\Sigma}\right) \mathrm{e}(t), \\
\mathcal{Z}^{\prime}=\left(\partial_{t}^{-1} \mathbf{P}^{\Lambda H}+\mathbf{P}^{\Sigma}\right) \mathcal{Z}\left(\mathbf{P}^{\Lambda H}+\partial_{t} \mathbf{P}^{\Sigma}\right) .
\end{gathered}
$$

The projectors $\mathbf{P}^{\Lambda H}$ and $\mathbf{P}^{\Sigma}$ are defined in [6], and project an arbitrary RWG coefficient vector onto the space of (local and global) RWG loops and onto the space of RWG stars, respectively. Equation (8) is the semi-discrete qHP-TDEFIE [3]. The system obtained from temporally discretizing (8) remains well-conditioned for large time steps, but not for dense spatial discretization.

Analogously to (9), define

$$
\tilde{\mathcal{Z}}^{\prime}=\left(\partial_{t}^{-1} \mathbb{P}^{\Sigma H}+\mathbb{P}^{\Lambda}\right) \tilde{\mathcal{Z}}\left(\mathbb{P}^{\Sigma H}+\partial_{t} \mathbb{P}^{\Lambda}\right),
$$

where the projectors $\mathbb{P}^{\Sigma H}$ and $\mathbb{P}^{\Lambda}$ project an arbitrary $\mathrm{BC}$ coefficient vector onto the space of (local and global) BC loops and onto the space of BC stars, respectively [6]. Analogously to (5), the following Calderón preconditioned equation is proposed:

$$
\left(\tilde{\mathcal{Z}}^{\prime} \mathbf{G}_{\mathrm{mx}}^{-1} \mathcal{Z}^{\prime} \mathrm{y}\right)(t)=-\left(\tilde{\mathcal{Z}}^{\prime} \mathbf{G}_{\mathrm{mx}}^{-1} e^{\prime}\right)(t),
$$

with

$$
\mathrm{e}^{\prime}(t)=\left(\partial_{t}^{-1} \mathbf{P}^{\Lambda H}+\mathbf{P}^{\Sigma}\right) \mathrm{e}(t)
$$

Note that (11) is not equivalent to (5). Upon temporal discretization, both equations will lead to a discrete system that is immune to dense discretization breakdown. However, only (11) will lead to a system that is low frequency stable when applied to multiply connected geometries. This will be shown in Section 4. But first, the temporal discretization procedure is detailed in Section 3.

\section{TEMPORAL DISCRETIZATION}

In [3], a low frequency and DC stable scheme was obtained by expanding $\mathrm{y}(t)$ as

$$
\mathrm{y}(t)=\sum_{i=1}^{N_{T}}\left(p(t-i \Delta t) \mathbf{P}^{\Lambda H}+h(t-i \Delta t) \mathbf{P}^{\Sigma}\right) \mathbf{y}^{i},
$$

where $p(t)$ are the pulse functions and $h(t)$ are the hat functions, as defined in [3]. The following auxiliary quantities are now proposed:

$$
\begin{aligned}
\mathrm{y}^{\prime}(t) & =\mathcal{Z}^{\prime} \mathrm{y}(t)+\mathrm{e}^{\prime}(t) \\
& \approx \sum_{i}\left(p(t-i \Delta t) \mathbf{P}^{\Sigma}+h(t-i \Delta t) \mathbf{P}^{\Lambda H}\right) \mathrm{y}_{i}^{\prime} \\
\mathrm{y}^{\prime \prime}(t) & =\mathbf{G}_{\mathrm{mx}}^{-1} \mathbf{y}^{\prime}(t) \\
& \approx \sum_{i}\left(p(t-i \Delta t) \mathbb{P}^{\Sigma H}+h(t-i \Delta t) \mathbb{P}^{\Lambda}\right) \mathrm{y}_{i}^{\prime \prime}
\end{aligned}
$$

In terms of these auxiliary unknowns, (11) becomes

$$
\tilde{\mathcal{Z}}^{\prime} \mathrm{y}^{\prime \prime}(t)=0 \text {. }
$$

In order to maintain compatibility with the scheme in [3], the following temporal Galerkin method is applied to (14):

$$
\begin{gathered}
\int_{\mathbb{R}}\left(\delta(t-j \Delta t) \mathbf{P}^{\Lambda H}+\frac{1}{\Delta t} p(t-j \Delta t) \mathbf{P}^{\Sigma}\right) \\
\cdot(\text { Equation (14)) } d t .
\end{gathered}
$$

A relation between $\mathrm{y}_{i}^{\prime \prime}$ and $\mathrm{y}_{j}^{\prime}$ is easily found using the following Galerkin method:

$$
\begin{gathered}
\int_{\mathbb{R}}\left(\delta(t-j \Delta t) \mathbb{P}^{\Lambda}+\frac{1}{\Delta t} p(t-j \Delta t) \mathbb{P}^{\Sigma H}\right) \\
\cdot(\text { Equation }(15)) d t,
\end{gathered}
$$


Finally the BC equivalent of (17) is applied to (16):

$$
\begin{gathered}
\int_{\mathbb{R}}\left(\delta(t-j \Delta t) \mathbb{P}^{\Sigma H}+\frac{1}{\Delta t} p(t-j \Delta t) \mathbb{P}^{\Lambda}\right) \\
\cdot(\text { Equation }(16)) d t .
\end{gathered}
$$

This all leads to an equation of the form

$$
\begin{aligned}
& \sum_{j} \sum_{k} \sum_{l} \mathbb{Z}_{j}^{\prime} \mathbf{G}_{k}^{-1} \mathbf{Z}_{l}^{\prime} \mathrm{y}^{i-j-k-l} \\
= & -\sum_{j} \sum_{k} \mathbb{Z}_{j}^{\prime} \mathbf{G}_{k}^{-1} \mathrm{e}^{i-j-k},
\end{aligned}
$$

where $\mathbf{Z}_{j}^{\prime}$ and $\mathrm{e}^{\prime j}$ are defined as in [3]. The definition of $\mathbb{Z}_{j}^{\prime}$ is similar to that of $\mathbf{Z}_{j}^{\prime}$, but uses BC functions instead of RWG functions. Finally,

$$
\begin{aligned}
\mathbf{G}_{0}^{-1}= & \frac{1}{2} \mathbb{P}^{\Sigma H} \mathbf{G}_{\mathrm{mx}}^{-1} \mathbf{P}^{\Lambda H}+\mathbb{P}^{\Sigma H} \mathbf{G}_{\mathrm{mx}}^{-1} \mathbf{P}^{\Sigma} \\
& +\mathbb{P}^{\Lambda} \mathbf{G}_{\mathrm{mx}}^{-1} \mathbf{P}^{\Lambda H} \\
\mathbf{G}_{1}^{-1}= & \frac{1}{2} \mathbb{P}^{\Sigma H} \mathbf{G}_{\mathrm{mx}}^{-1} \mathbf{P}^{\Lambda H}
\end{aligned}
$$

Equation (20) can be solved using the MOT algorithm, and remains well-conditioned for dense meshes. Also the MOT system matrix $\mathbb{Z}_{0}^{\prime} \mathbf{G}_{0}^{-1} \mathbf{Z}_{0}^{\prime}$ remains well-conditioned, as will be shown in Section 4. This observation suggests the use of the matrix $\mathbb{Z}_{0}^{\prime} \mathbf{G}_{0}^{-1}$ as a purely multiplicative preconditioner for the qHP-TDEFIE. The preconditioned MOT equation then becomes

$$
-\mathbb{Z}_{0}^{\prime} \mathbf{G}_{0}^{-1} \mathbf{Z}_{0}^{\prime} \mathrm{y}^{j}=\mathbb{Z}_{0}^{\prime} \mathbf{G}_{0}^{-1}\left(\sum_{i=1}^{j} \mathbf{Z}_{i}^{\prime} \mathrm{y}^{j-i}+\mathrm{e}^{\prime j}\right) .
$$

Just like the qHP-TDEFIE [3], equation (23) is immune to both DC instability and low frequency breakdown, on simply as well as multiply connected geometries. Additionally, it is is immune to dense discretization breakdown.

The proposed equation (23) has several advantages over the dottrick TD-EFIE [1]. First, the preconditioner $\mathbb{Z}_{0}^{\prime} \quad \mathbf{G}_{0}^{-1}$ is purely multiplicative, and can be applied directly to the original qHPTDEFIE. Second, (23) correctly handles global topological loops, whereas the dottrick TD-EFIE does not. As a result, the dottrick TD-EFIE suffers from DC instability [2] and low frequency breakdown [3] when applied to multiply connected geometries.

\section{NUMERICAL RESULTS}

Consider the perfectly conducting double torus in Fig. 1, which is obtained by merging two tori with large radius $1 \mathrm{~m}$ and small radius $0.4 \mathrm{~m}$. Transient scattering by this object is simulated using the following formulations:

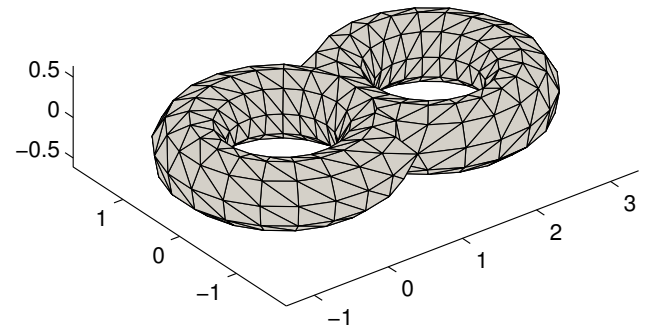

Figure 1: Mesh of a double torus. The large radius of each torus is $1 \mathrm{~m}$, and the small radius $0.4 \mathrm{~m}$. The triangle mesh contains 692 triangles and its mesh parameter $h=0.32 \mathrm{~m}$.



Figure 2: The condition number of the MOT system matrix for different formulations, as a function of the time step $\Delta t$.

- the standard TD-EFIE,

- the qHP-TDEFIE [3],

- the dottrick TD-EFIE [1],

- the Calderón preconditioned qHP-TDEFIE or CP qHP-TDEFIE (23).

Each of these methods is applied to the mesh shown in Fig. 1, with mesh parameter $h=0.32 \mathrm{~m}$. The time step is varied form $c \Delta t=1 \mathrm{~m}$ to $c \Delta t=$ $1024 \mathrm{~m}$. The condition number of the MOT system matrix (i.e., $\mathbf{Z}_{0}, \mathbf{Z}_{0}^{\prime}, \mathbb{Z}_{0}^{\prime} \mathbf{G}_{0}^{-1} \mathbf{Z}_{0}^{\prime}$ or equivalent) is shown in Fig. 2. The TD-EFIE and dottrick TD-EFIE simulations exhibit low frequency breakdown: the condition number of their MOT system matrices grow proportionally to $\Delta t^{2}$. The qHP-TDEFIE and CP qHP-TDEFIE on the other hand remain well-conditioned regardless of the chosen time step.

Next, the time step is kept constant at $c \Delta t=4 \mathrm{~m}$ while the mesh parameter $h$ is varied from $0.16 \mathrm{~m}$ to $0.42 \mathrm{~m}$. Again, the condition number of the MOT 


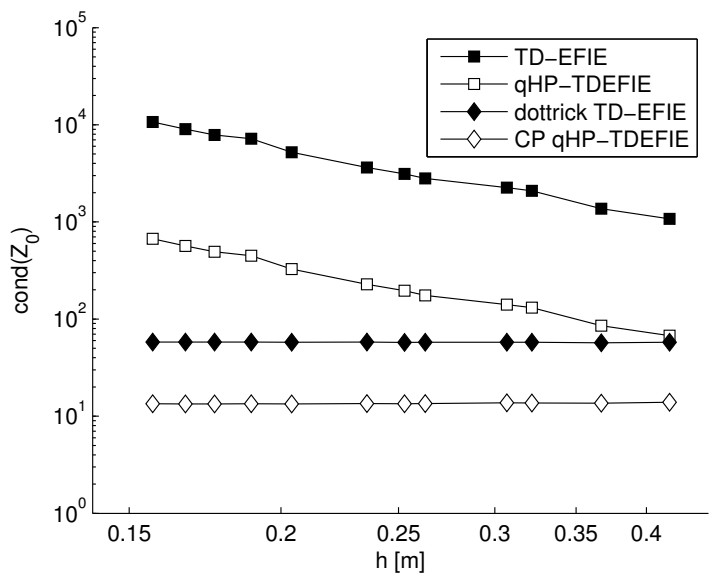

Figure 3: The condition number of the MOT system matrix for different formulations, as a function of the mesh parameter $h$.

system matrix is plotted in Fig. 3. The TD-EFIE and qHP-TDEFIE simulations suffer from dense discretization breakdown: the condition number of their MOT system matrices grow proportionally to $h^{-2}$. The dottrick TD-EFIE and CP qHP-TDEFIE schemes remain well-conditioned even for dense spatial discretizations.

This example shows that for a multiply connected geometry like the double torus in Fig. 1, only the CP qHP-TDEFIE scheme (20) remains wellconditioned both for large time steps and for dense meshes.

\section{CONCLUSIONS}

In this contribution, a multiplicative preconditioner for the qHP-TDEFIE was developed. The resulting preconditioned scheme is immune to both low frequency breakdown and dense discretization breakdown, and is therefore able to efficiently model transient scattering problems involving slowly varying fields as well as geometries with fine geometrical details. In contrast to the dottrick TD-EFIE, this approach is also applicable to multiply connected geometries. Furthermore, it can be directly applied to the qHP-TDEFIE without requiring additional changes to the algorithm.

\section{Acknowledgments}

The work of Y. Beghein was supported by a doctoral grant from the Agency for Innovation by Science and Technology in Flanders (IWT).

\section{References}

[1] K. Cools, F. Andriulli, F. Olyslager, and E. Michielssen, "Time domain Calderón identities and their application to the integral equation analysis of scattering by PEC objects part I: Preconditioning," IEEE Transactions on Antennas and Propagation, vol. 57, no. 8, pp. 2352-2364, Aug 2009.

[2] — , "Nullspaces of MFIE and Calderón preconditioned EFIE operators applied to toroidal surfaces," IEEE Transactions on Antennas and Propagation, vol. 57, no. 10, pp. 3205-3215, Oct 2009.

[3] Y. Beghein, K. Cools, and F. Andriulli, "A DC stable and large time step well-balanced TDEFIE based on quasi-Helmholtz projectors," IEEE Transactions on Antennas and Propagation, vol. 63, no. 7, July 2015.

[4] N.-W. Chen, K. Aygun, and E. Michielssen, "Integral-equation-based analysis of transient scattering and radiation from conducting bodies at very low frequencies," Microwaves, Antennas and Propagation, IEE Proceedings, vol. 148, no. 6, pp. 381-387, Dec 2001.

[5] F. Andriulli, "Loop-star and loop-tree decompositions: Analysis and efficient algorithms," IEEE Transactions on Antennas and Propagation, vol. 60, no. 5, pp. 2347-2356, May 2012.

[6] F. Andriulli, K. Cools, I. Bogaert, and E. Michielssen, "On a well-conditioned electric field integral operator for multiply connected geometries," IEEE Transactions on Antennas and Propagation, vol. 61, no. 4, pp. 2077-2087, April 2013.

[7] S. Rao, D. Wilton, and A. Glisson, "Electromagnetic scattering by surfaces of arbitrary shape," IEEE Transactions on Antennas and Propagation, vol. 30, no. 3, pp. 409 - 418, may 1982.

[8] A. Buffa and S. H. Christiansen, "A dual finite element complex on the barycentric refinement," Comptes Rendus Mathematique, vol. 340, no. 6, pp. $461-464,2005$. 